\title{
Unexpected properties of the inductively coupled plasma induced defect in germanium
}

\author{
S. M. M. Coelho*, F. D. Auret, P. J. Janse van Rensburg, J. M. Nel \\ Department of Physics, University of Pretoria, Private Bag X20, Hatfield, 0028, South Africa. \\ *Corresponding author e-mail address: sergio@up.ac.za
}

\begin{abstract}
Inductively coupled plasma (ICP) etching of germanium introduces a single defect, the $\mathrm{E}_{0.31}$ electron trap, for a large range of argon partial pressures from $4 \times 10^{-3}$ to $6.5 \times 10^{-4} \mathrm{mbar}$ that correspond to ion energies of 8 to 60 $\mathrm{eV}$. Ge of three crystallographic orientations, (100), (110) and (111), treated with 20 and $60 \mathrm{eV} \mathrm{ICP} \mathrm{had} \mathrm{defect}$ concentration profiles that were similar in appearance, with a maximum concentration of $10^{14} \mathrm{~cm}^{-3}$ extending more than a $\mu \mathrm{m}$ into the material, approximately three orders of magnitude deeper than what TRIM simulations predicted. All profiles were measured using Laplace deep level transient spectroscopy (L-DLTS), a technique that is sensitive to defect concentrations as low as $10^{11} \mathrm{~cm}^{-3}$. Isochronal annealing of samples showed concentration curves broadening after a $400 \mathrm{~K}$ anneal and decreasing to the $10^{13} \mathrm{~cm}^{-3}$ level after a $450 \mathrm{~K}$ anneal. Unannealed samples measured after a year exhibited similar decreases in defect concentration without broadening of their profiles. A $550 \mathrm{~K}$ anneal lowered the defect concentration to levels below the L-DLTS detection limit. Thereafter additional plasma treatment of the surface failed to reintroduce this defect indicating that the structure required for the formation of $\mathrm{E}_{0.31}$ was no longer present in the region under observation.
\end{abstract}

Keywords: Germanium, DLTS, defect, anneal, diffusion

PACS: 81.05.Cy, 52.80.Pi, 61.72.Cc, 61.72.-y, 66.30.Lw, 61.72.Cc

\section{Introduction}

Germanium was the semiconductor used to demonstrate the first transistor and continues to show promise as a suitable candidate to use in ultra-fast devices. It remains the best material for gamma ray detectors and far infrared detectors [1]. Process induced defects are unfortunately introduced during device manufacture; such defects either improve device performance [2] or, as in the case of photo-voltaics, impair their function [3]. Sputter etching is a key technology in the manufacture of semiconductor devices with RF sputter etching and deposition being the most popular in this industry as large wafers can be processed uniformly [4]. With low energy ions producing low ion damage [4,5], high plasma density and the availability of large area sources, inductively coupled plasma (ICP) etching may in future displace capacitively coupled RF plasma sources as the technology of choice to etch large wafers.

Argon ICP etching introduces only one defect in $\mathrm{Ge}[6], \mathrm{E}_{0.31}$, with an energy level $\mathrm{E}_{\mathrm{c}}-0.31 \mathrm{eV}$ and an apparent capture cross-section of $3.5 \times 10^{-14} \mathrm{~cm}^{2}$. This defect has not been observed after MeV electron irradiation [7,8], alpha particle irradiation [9], sputter deposition [10] or electron beam deposition [11] but was observed after dchydrogen plasma passivation [12]. Comparing DLTS spectra, the $\mathrm{E}_{0.31}$ defect reported on in sputter deposition [10] exhibits a peak at $150 \mathrm{~K}$ at a rate window of $80 \mathrm{~s}^{-1}$ whereas the ICP induced defect has a peak at $157 \mathrm{~K}$. The Arrhenius plots of these two defects further confirmed that they are different defects. The inertness of argon and the low energy of the ICP Ar ions (measured by the source manufacturer using a Faraday cup) make it unlikely that $\mathrm{E}_{0.31}$ would be introduced during high energy processes where conditions are very different. The reproducible introduction of a single defect provides the ideal platform to study the effect of this defect on the properties of a well-known semiconductor. Our focus for this work was to observe the introduction of $\mathrm{E}_{0.31}$ in ultra-pure n-type Ge crystals with crystallographic orientations (100), (110) and (111), all bulk grown by Umicore but with differing Sb concentrations of $9 \times 10^{14}, 2.3 \times 10^{14}$ and $1.3 \times 10^{15} \mathrm{~cm}^{-3}$ respectively. Samples, one of each crystallographic orientation, were processed simultaneously and thereafter Laplace deep level transient spectroscopy (L-DLTS) [13] was used to obtain the defect concentration profiles of $\mathrm{E}_{0.31}$ in our samples.

\section{Experimental procedure}

Electronic grade polished Ge wafers with carbon and oxygen impurity concentrations lower than $10^{16} \mathrm{~cm}^{-3}$ (measured using SIMS) of three crystallographic orientations, (100), (110) and (111), were diced into $3 \times 5 \mathrm{~mm}$ pieces. These were subsequently degreased in successive five minute ultrasonic baths of trichloroethylene, isopropanol and methanol followed by a one minute etch in $1: 5, \mathrm{H}_{2} \mathrm{O}_{2}: \mathrm{H}_{2} \mathrm{O}$. AuSb $(0.6 \% \mathrm{Sb})$ was evaporated 
resistively onto the back surface of all the samples and annealed at $625 \mathrm{~K}$ for ten minutes in Ar to lower the resistance of these contacts thus making them ohmic. The same wet cleaning procedure was followed prior to loading one sample of each crystallographic orientation into the vacuum chamber for ICP etching using a Copra 160 source from CCR Technology. This source produces an ion energy distribution curve with two narrow peaks with a full width half maximum that is less than $1 / 3$ of the maximum energy. The quoted values herein are for the high energy peaks. The ICP system chamber was pumped down to $1 \times 10^{-6} \mathrm{mbar}$ before being backfilled with Ar up to a partial pressure of $6.5 \times 10^{-4}$ mbar or $1 \times 10^{-3}$ mbar for the 3 minutes or 10 minutes ICP treatments respectively at $550 \mathrm{~W}$ plasma power and $1.5 \mathrm{~A}$ coil current. Plasma etching was followed by resistive evaporation (RE) of a $25 \mathrm{~nm}$ thick Pd layer through a stainless steel contact mask thus forming eight Schottky barrier diodes $0.6 \mathrm{~mm}$ in diameter on the front surface of each sample. RE is known to not introduce defects in quantities measurable by DLTS but diodes prepared by electron beam deposition (EBD) after ICP would also be suitable as no additional defects were observed in such samples.

Current-voltage (IV) and capacitance-voltage (CV) measurements were performed at room temperature to establish the ideality factor, reverse bias current at $1 \mathrm{~V}$, carrier concentration and built-in voltage $\mathrm{V}_{\mathrm{bi}}$ of the diodes. Only diodes with an ideality below 1.2 and a reverse bias current below $3 \times 10^{2} \mathrm{~A} \mathrm{~cm}^{-2}$ were considered suitable for further investigation. Applying conventional DLTS confirmed that $\mathrm{E}_{0.31}$ was introduced and thereafter L-DLTS was used to determine the peak amplitude at a fixed temperature while varying the filling pulse from $0.1 \mathrm{~V}$ to $5 \mathrm{~V}$. In fig. 1 the DLTS peak amplitudes can be converted to concentration of deep levels, $\mathrm{N}_{\mathrm{T}}$, as $\frac{N_{T}}{N_{D}}=\frac{2 \Delta C}{C}$ where $\mathrm{N}_{\mathrm{D}}$ is the concentration of shallow impurities, $\Delta \mathrm{C}$ is the DLTS peak height and $\mathrm{C}$ is the junction capacitance. To calculate the defect concentrations plotted in figures 2, 3 and 4 the approach of Zohta and Watanabe was used [14].

Samples were isochronally annealed for 10 minutes at $375 \mathrm{~K}, 400 \mathrm{~K}, 450 \mathrm{~K}, 500 \mathrm{~K}$ and $550 \mathrm{~K}$ in an Ar ambient and measured (IV and CV) after each annealing cycle. Long-term study samples were kept at room temperature (RT) between measurements.

\section{Results and discussion}

ICP etching introduced a single defect in the three crystals under consideration (fig. 1): the $\mathrm{E}_{0.31}$ electron trap. This observation held for ICP treatments at Ar pressures ranging from $4 \times 10^{-3}$ to $6.5 \times 10^{-4}$ mbar with corresponding ion energies from 8 to $60 \mathrm{eV}$. Although the ion current density was fairly constant at low Ar pressures it decreases linearly from $0.35 \mathrm{~mA} \mathrm{~cm}{ }^{-2}$ if the pressure increases above $10^{-3} \mathrm{mbar}$, leading to a corresponding drop in defect density with rising pressure. The DLTS spectra in fig. 1 of $60 \mathrm{eV}$ plasma etched diodes were obtained using a reverse bias voltage $\mathrm{V}_{\mathrm{r}}=-1 \mathrm{~V}$, and pulse $\mathrm{V}_{\mathrm{p}}=1.2 \mathrm{~V}$, these being suitable parameters to probe Ge close to the interface while not activating electron traps that lie deeper in the material. It is significant that near the metal-semiconductor interface the defect concentration in Ge (111) was substantially higher than that in the (100) and (110) samples. TRIM simulations of $60 \mathrm{eV}$ Ar ion bombardment predicted that the majority of ions would come to rest within $3 \mathrm{~nm}$ of the surface but did not take channeling or defect motion / diffusion into account. As the (111) crystal face presents the smallest channels to incident perpendicular ions followed by the (100) and (110) faces it is reasonable to expect that more damage occurs nearer to the surface of the (111) material. This is confirmed by the depth profiles in fig. 2 up to a depth of $0.5 \mu \mathrm{m}$, however, by $0.6 \mu \mathrm{m}$ depth below the junction the defect concentration was similar for all three samples. At Ar ion energy of $20 \mathrm{eV}$ (fig. 4, hollow symbols) no significant differences were observed for defect concentrations near the interface.

The 3 minute $60 \mathrm{eV}$ plasma treatment introduced $\mathrm{E}_{0.31}$ in all three crystals observable up to a depth of almost 2 $\mu \mathrm{m}$. Beyond a depth of $0.6 \mu \mathrm{m}$ all samples exhibited a log-linear decrease in concentration with increasing depth below the junction with no clearly discernible differences between crystal orientations. The $\mathrm{E}_{0.31}$ concentration may decline slightly more with increasing depth in $\mathrm{Ge}(111)$, however, these differences in fig. 2 and fig. 4 may also be ascribed to experimental error. A $375 \mathrm{~K}$ anneal had a small effect on the defect profiles with the defect profile moving approximately $0.1 \mu \mathrm{m}$ into the material. A $400 \mathrm{~K}$ anneal produced marked changes in the defect profiles with an overall profile broadening and defect concentration lowering as $\mathrm{E}_{0.31}$ diffuses deeper into the material, the concentration near the surface reducing by more than $50 \%$. The defect concentration in $\mathrm{Ge}(111)$ remained substantially higher than the concentration in the other crystals in the first $\mu \mathrm{m}$ below the junction and could not be measured deeper into the material due to limitations of the pulse generator. A similar decrease in defect concentration was observed in the measured volume after the $450 \mathrm{~K}$ anneal except for the (111) sample whose defect concentration fell more sharply resulting in it being only slightly higher than the (110) and (100) profiles. A further broadening of the defect concentration profile occurred after the $450 \mathrm{~K}$ anneal. A $500 \mathrm{~K}$ anneal drove the defect concentration down dramatically to the $10^{12}$ defects $\mathrm{cm}^{-3}$ level in the (111) and (110) samples and even lower for the (100) sample. The reason that the defect concentration in the (100) sample dropped more rapidly is unclear as the samples were always processed together. A $550 \mathrm{~K}$ anneal lowered the defect concentration below the measurement threshold of our equipment. 


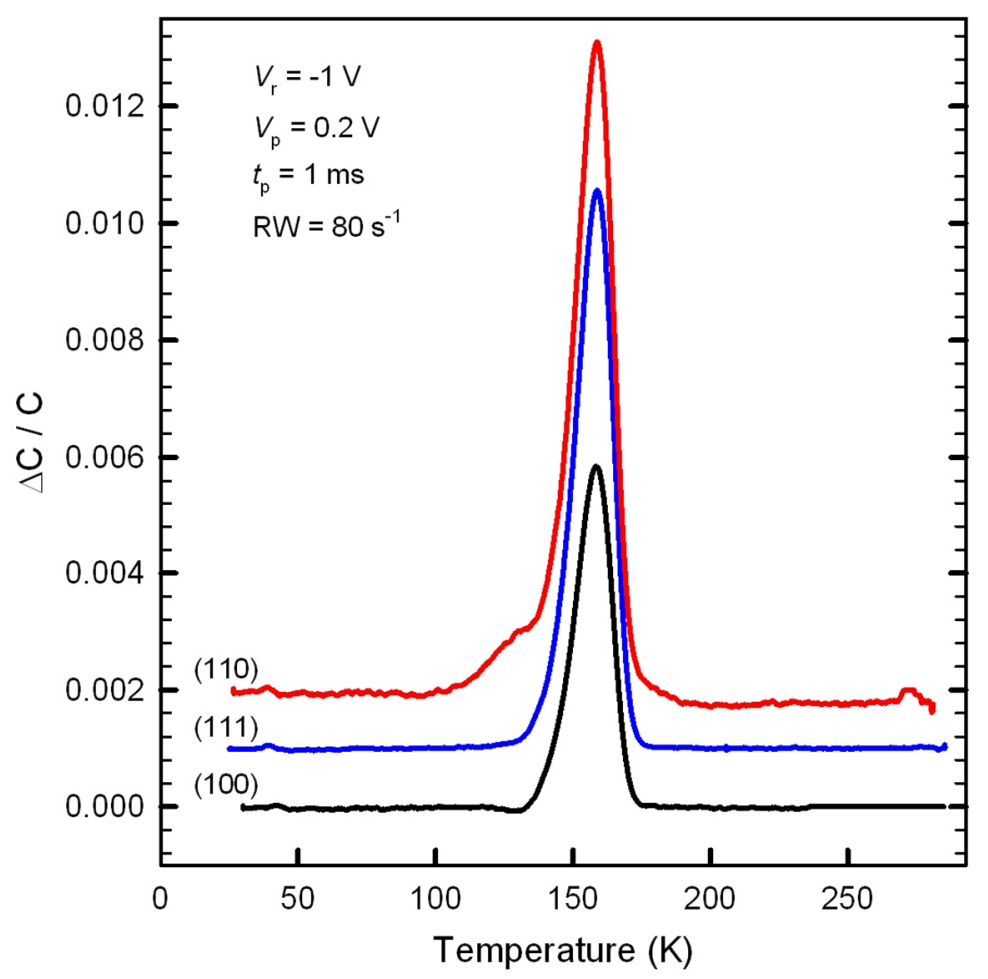

Fig. 1. DLTS spectra of resistively evaporated Pd diodes on ICP etched Ge $(1,0,0),(1,1,0)$ and $(1,1,1)$. The three samples were coprocessed and received 3 minutes of ICP etching. Spectra were recorded at a rate window of $80 \mathrm{~s}^{-1}$, pulse width of $1 \mathrm{~ms}$, quiescent reverse bias of $-1 \mathrm{~V}$ and a filling pulse with an amplitude of $1.2 \mathrm{~V}$. To distinguish between spectra the (111) and the (110) plots have been offset by 0.001 and 0.002 along the $y$-axis, respectively. Some measurements exhibit higher background noise but this did not influence the results.

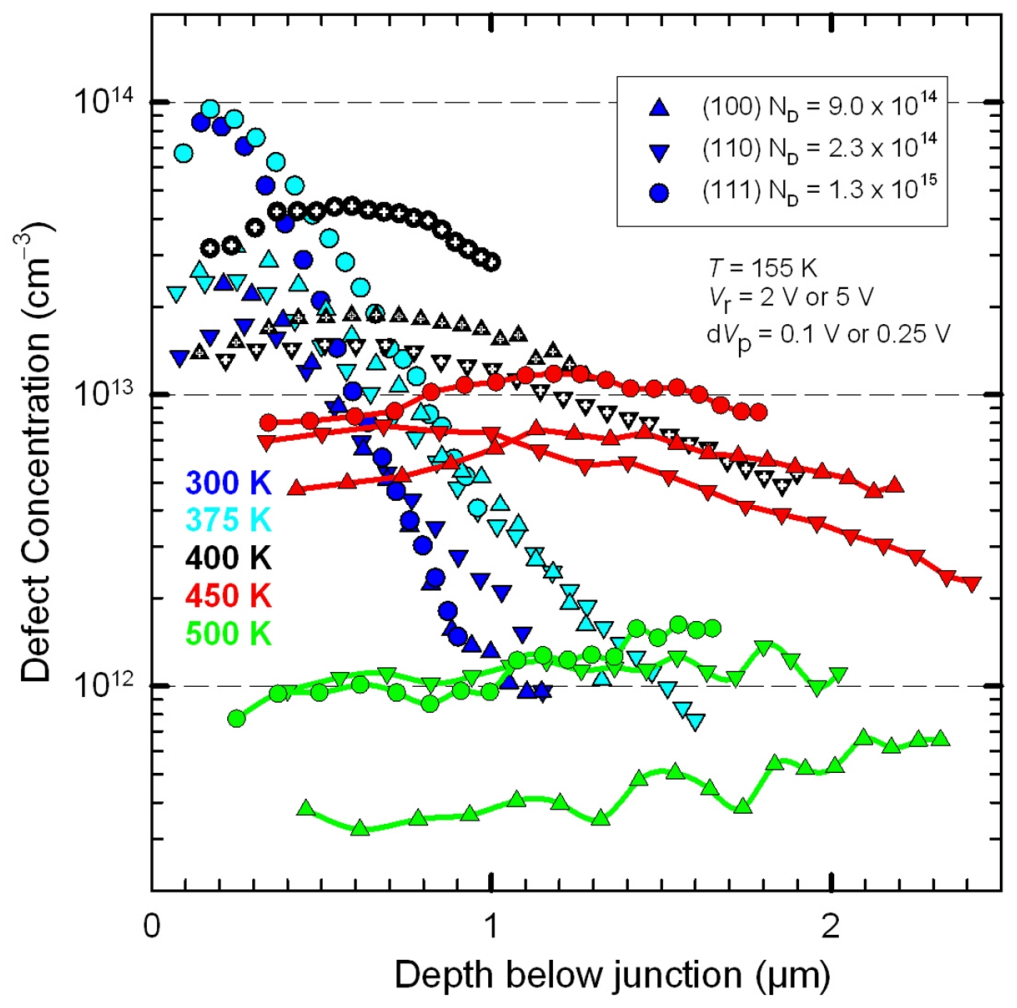

Fig. 2. Depth profiles of the ICP induced $E_{0.31}$ defect in $\mathrm{Ge}(1,0,0),(1,1,0)$ and $(1,1,1)$ recorded directly after 3 minute $\mathrm{ICP}$ etching at an Ar partial pressure of $7 \times 10^{-4}$ mbar and diode fabrication with subsequent measurements taken after 10 minute isochronal annealing at $375 \mathrm{~K}$, $400 \mathrm{~K}, 450 \mathrm{~K}$ and $500 \mathrm{~K}$ respectively. Peak amplitudes for pulses varying from $0.1 \mathrm{~V}$ to $2 \mathrm{~V}$ or $5 \mathrm{~V}$ were determined using L-DLTS. 


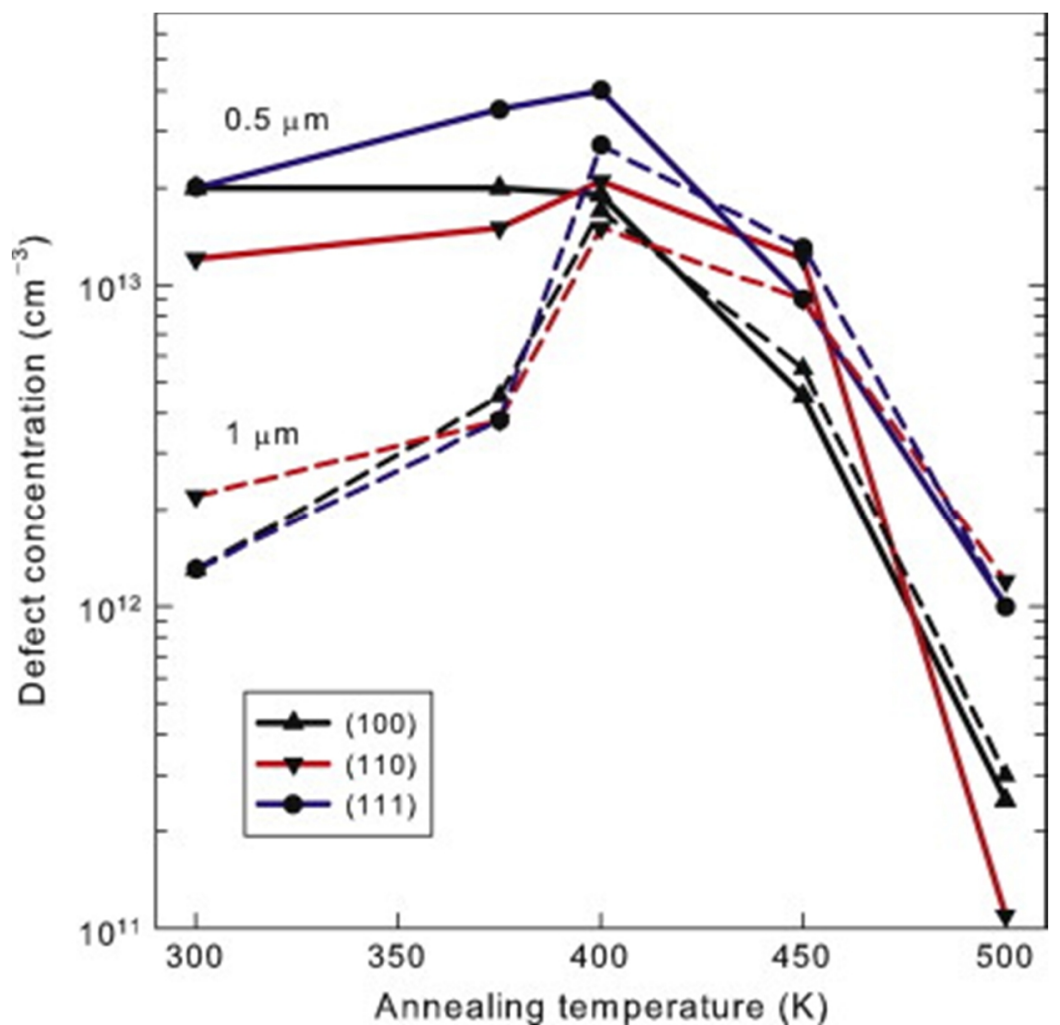

Fig. 3. A summary of fig. 2, plotting defect concentration versus annealing temperature at $0.5 \mu \mathrm{m}$ and $1 \mu \mathrm{m}$ below the metal-semiconductor interface respectively.

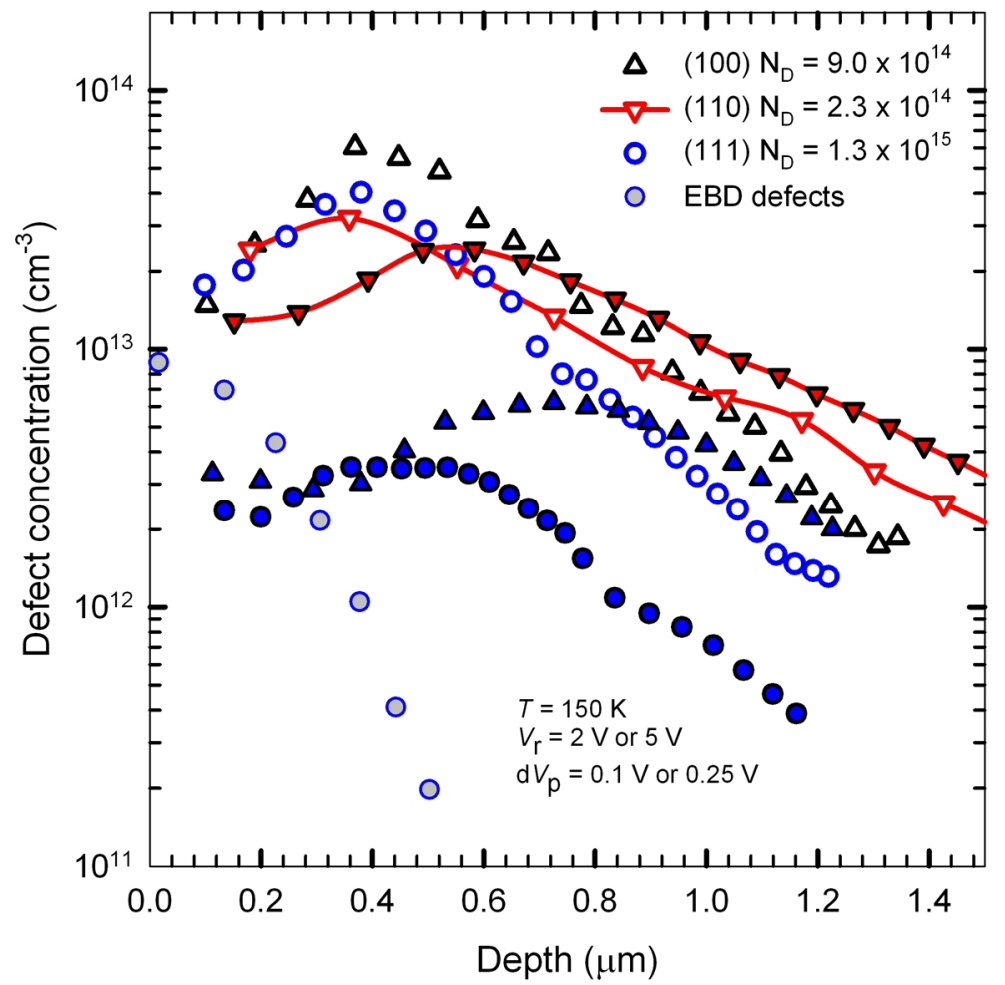

Fig. 4. Depth profiles of the $\mathrm{E}_{0.31}$ defect in $\mathrm{Ge}(1,0,0),(1,1,0)$ and $(1,1,1)$ recorded directly after a 10 minute ICP etch at an Ar partial pressure of $10^{-3} \mathrm{mbar}$ and diode fabrication (hollow symbols). The co-processed samples were kept at room temperature and measured again after one year (solid symbols). Peak amplitudes for pulses varying from $0.1 \mathrm{~V}$ to $2 \mathrm{~V}$ or $5 \mathrm{~V}$ were determined using L-DLTS. The total concentration of EBD induced electron traps recorded immediately after diode deposition is shown for comparison purposes. 
The net carrier concentration extracted from CV measurements performed at room temperature did not vary by more than $5 \%$ when comparing control diodes to ICP treated diodes and thus did not have a significant impact on the defect depth profiles that were calculated. The depth profiles of the net carrier concentration were constant from a depth of $0.9 \mu \mathrm{m}$ into the material with a lowering in concentration towards the surface. There were no noticeable changes in the capacitance measured as the samples were annealed at successively higher temperatures.

A summary of fig. 2 was plotted for the defect concentration at $0.5 \mu \mathrm{m}$ and $1 \mu \mathrm{m}$ below the junction (fig. 3). These depths were chosen as they correspond to readings taken where $\mathrm{V}_{\mathrm{p}}$ is large resulting in strong DLTS signals thus improving the measurement accuracy. At both depths below the surface all diodes exhibited the same trends independent of crystal orientation and majority carrier, $\mathrm{Sb}$, concentration. A number of defects in Ge have been shown to be related to $\mathrm{Sb}$, such as the E-center, but $\mathrm{E}_{0.31}$ shows no dependence on the $\mathrm{Sb}$ concentration and is unlikely to be related to the $\mathrm{Sb}$ dopant.

Finally, samples ICP etched for 10 minutes at an Ar partial pressure of $10^{-3}$ mbar, coinciding with ICP ion energy of $20 \mathrm{eV}$, were measured and then measured again after a period of a year. The depth profiles are plotted in fig. 4 together with the cumulative depth profile of the major EBD induced electron traps in Ge. The initial plots (fig. 4, hollow symbols) are very similar to those plotted for the samples in fig. 2 before annealing. Measurement of the (111) sample showed no change after 8 weeks (not plotted) but after one year there is a decrease in concentration of almost an order of magnitude up to a depth of $0.5 \mu \mathrm{m}$. Changes in the (100) and (110) samples were not as dramatic but all samples exhibited a decrease in concentration and diffusion deeper into the material after a year. In contrast to the samples that were annealed, these samples do not display a broadening of their defect concentration profiles with time even though in the case of the (100) and (111) samples the defect concentration fell to levels similar to those observed after the $450 \mathrm{~K}$ anneal suggesting that a different process is responsible for these changes, a process that is not dependent on the normal diffusion of this particular defect into the bulk.

The ICP induced defect is also distributed far deeper into the material and in greater concentration than the accumulated defects of a typical $10 \mathrm{keV}$ EBD evaporation and it was previously observed that if the $\mathrm{E}_{0.31}$ defect is present then no additional defects are introduced in measurable quantities during EBD [15]. The available defect sites, when occupied by the $\mathrm{E}_{0.31}$ defect remain unavailable to EBD induced defects. An attempt to reintroduce the $\mathrm{E}_{0.31}$ defect after a $550 \mathrm{~K}$ anneal by ICP etching the sample again and evaporating diodes onto the etched surface was unsuccessful indicating that once the physical system that was required for the ICP defect to be created has been annealed to an undetectable level, no physical systems remain for additional defects to form. This suggests that the same site required for the formation of $\mathrm{E}_{0.31}$ is also necessary for the formation of EBD induced defects in ultra-pure Ge. As hydrogen plasma passivation was found to introduce $\mathrm{E}_{0.31}$ it was postulated that this defect may be hydrogen related [12], however, as Nyamhere et al further found that $\mathrm{E}_{0.31}$ was also introduced using He plasma, a conclusion was drawn that this defect is not related to $\mathrm{H}$ [16] but this is incorrect as there was no determination of whether $\mathrm{H}$ was present in the samples prior to treatment or whether the treatment eliminated $\mathrm{H}$ from the sample. On the contrary, it has been known for many years that $\mathrm{H}$ remains trapped in the crystal in concentrations of $10^{14}$ to $10^{15} \mathrm{~cm}^{-3}$ when it is cooled from the melting point to room temperature (see [17] and reference 4 therein). Hydrogen in germanium has not been as well studied as hydrogen in silicon [18] but it is expected that $\mathrm{V}_{\mathrm{m}} \mathrm{H}_{\mathrm{n}}$ complexes also form in Ge, wherein $\mathrm{VH}_{2}, \mathrm{VH}_{4}$ and $\mathrm{V}_{2} \mathrm{H}_{6}$ were identified using infrared (IR) absorption studies [19]. At present no hydrogen related defects have been identified in Ge using DLTS, however, $\mathrm{VH}_{1}$ and $\mathrm{VH}_{3}$ have been predicted to each have a deep level between the valence and conduction bands [20]. IR lines ascribed to $\mathrm{VH}_{2}$ and $\mathrm{VH}_{4}$ disappeared after annealing at $400 \mathrm{~K}$ and $590 \mathrm{~K}$ respectively [21] and as $\mathrm{VH}_{3}$ diffuses at a lower temperature than $\mathrm{VH}$ in silicon [22] it was expected that annealing the sample to $550 \mathrm{~K}$ would diffuse $\mathrm{VH}_{3}$ into the bulk but not diffuse $\mathrm{VH}_{4}$ back towards the region under the junction which is one possible explanation of why additional ICP treatment failed to reintroduce this defect. The identification of the $\mathrm{E}_{0.31}$ defect as $\mathrm{VH}_{3}$ is speculative at this time but the lack of other impurities suggests that hydrogen plays a role in the formation of this defect. $\mathrm{VH}_{1}$ is not a suitable candidate as its annealing temperature would have to be lower than the annealing temperature of $\mathrm{VH}_{2}(400 \mathrm{~K})$. All the processes that introduced $\mathrm{E}_{0.31}$ were similar in that the Ge surface was bombarded with ions of energy between $20 \mathrm{eV}$ and $100 \mathrm{eV}$ yet the upper and lower bounds for the introduction of this defect remain to be established.

\section{Conclusion}

$E_{0.31}$ was introduced by ICP etching up to a maximum concentration of $9 \times 10^{13} \mathrm{~cm}^{-3}$ at similar concentrations for all crystal orientations, independent of Sb concentration. It is unlikely that the ICP defect is Sb related and as Ge is known to have low oxygen and carbon impurity levels, these too are unlikely candidates. Hydrogen, or its removal, was found to be the most likely element responsible for the introduction of $\mathrm{E}_{0.31}$. Defects were detectable a few microns into the samples, almost 1000 times deeper than predicted by TRIM simulations of Ar implantation into Ge. Defect diffusion and tunneling of Ar ions may play a small role in explaining the 
unannealed profiles but cannot completely account for these defect distributions considering the depth of defect position and the differences in profile shape between aged samples and annealed samples. ICP was unable to reintroduce $\mathrm{E}_{0.31}$ after it had been annealed to undetectable levels. The first plasma treatment proved to be a very efficient process to convert the majority of available sites into $\mathrm{E}_{0.31}$ defect sites while the second ICP etch failed to find new sites to convert. The process by which ICP introduces defects deep into the material is not well understood and merits further study.

\section{Acknowledgements}

The authors are grateful for the financial support extended to them by the South African National Research Foundation. Special thanks are due to A. R. Peaker (Centre for Electronic Materials Devices and Nanostructures, University Of Manchester) and L. Dobaczewski (Institute of Physics, Polish Academy of Science) for supplying the Laplace software and hardware used in this study.

\section{References}

[1] E.E. Haller, Germanium: From its discovery to SiGe devices, Materials Science in Semiconductor Processing. 9 (2006) $408-422$.

[2] D. Sawko, J. Bartko, Production of fast switching power thyristors by proton irradiation, IEEE Transactions on Nuclear Science. NS-30 (1982) 1756-1758.

[3] S.Z. Karazhanov, Mechanisms for the anomalous dependence of carrier lifetime on injection level and photoconductance on light intensity, Journal of Applied Physics. 89 (2001) 332-335.

[4] T. Chevolleau, W. Fukarek, Ion flux, ion energy distribution and neutral density in an inductively coupled argon discharge, Plasma Sources Science and Technology. 9 (2000) 568-573.

[5] Z. Ren, M. Dineen, L. Deng, A.L. Goodyear, R. Gunn, Large batch etching of gallium nitride using Inductively Coupled Plasma tools as a production solution, Materials Research Society Symposium Proceedings. 1195 (2010) 115-120.

[6] F.D. Auret, S.M.M. Coelho, G. Myburg, P.J.J. Van Rensburg, W.E. Meyer, Defect introduction in Ge during inductively coupled plasma etching and Schottky barrier diode fabrication processes, Thin Solid Films. 518 (2010) 2485-2488.

[7] N. Fukuoka, H. Saito, Defect States in n-Type Germanium Irradiated With 1. 5 MeV Electrons, Jpn. J. Appl. Phys. 1. V 21 (1982) 930-935.

[8] J. Fage-Pedersen, A. Nylandsted Larsen, A. Mesli, Irradiation-induced defects in Ge studied by transient spectroscopies, Physical Review B. 62 (2000) 10116-10125.

[9] Vl. Kolkovsky, M.C. Petersen, A.N. Larsen, Alpha-particle irradiation-induced defects in n-type germanium, Applied Physics Letters. 90 (2007) 112110.

[10] F.D. Auret, S. Coelho, W.E. Meyer, C. Nyamhere, M. Hayes, J.M. Nel, Electrical Characterization of Defects Introduced During Sputter Deposition of Schottky Contacts on n-type Ge, Journal of Electronic Materials. 36 (2007) 1604-1607.

[11] F.D. Auret, S.M.M. Coelho, M. Hayes, W.E. Meyer, J.M. Nel, Electrical characterization of defects introduced in Ge during electron beam deposition of different metals, Physica Status Solidi (a). 205 (2008) 159-161.

[12] C. Nyamhere, A. Venter, D.M. Murape, F.D. Auret, S.M.M. Coelho, J.R. Botha, dc-Hydrogen plasma induced defects in bulk nGe, Physica B: Condensed Matter. 407 (2012) 2935-2938.

[13] L. Dobaczewski, P. Kaczor, Laplace transform deep-level transient spectroscopic studies of defects in semiconductors, Journal of Applied Physics. 76 (1994) 194-198.

[14] Y. Zohta, M.O. Watanabe, On the determination of the spatial distribution of deep centers in semiconducting thin films from capacitance transient spectroscopy, Journal of Applied Physics. 53 (1982) 1809-1811.

[15] F.D. Auret, S.M.M. Coelho, G. Myburg, P.J. Janse van Rensburg, W.E. Meyer, Electronic and annealing properties of the E0.31 defect introduced during Ar plasma etching of germanium, Physica B: Condensed Matter. 404 (2009) 4376-4378.

[16] C. Nyamhere, A. Venter, F.D. Auret, S.M.M. Coelho, D.M. Murape, Characterization of the E(0.31) defect introduced in bulk nGe by H or He plasma exposure, Journal of Applied Physics. 111 (2012) 044511 
[17] J. Weber, M. Hiller, E.V. Lavrov, Hydrogen in germanium, Materials Science in Semiconductor Processing. 9 (2006) $564-570$.

[18] A.R. Peaker, V.P. Markevich, L. Dobaczewski, Defects in Microelectronic Materials and Devices, CRC, New York, 2009.

[19] M. Budde, B. Bech Nielsen, J.C. Keay, L.C. Feldman, Vacancy hydrogen complexes in group-IV semiconductors, Physica B: Condensed Matter. 273-274 (1999) 208-211.

[20] B.J. Coomer, P. Leary, M. Budde, B. Bech Nielsen, R. Jones, S. Öberg, et al., Vacancy-hydrogen complexes in germanium, Materials Science and Engineering: B. 58 (1999) 36-38.

[21] M. Budde, Hydrogen-related defects in proton-implanted silicon and germanium. An Infrared Absorption Study, PhD thesis, University of Aarhus, 1998.

[22] S.K. Estreicher, A. Docaj, M.B. Bebek, D.J. Backlund, M. Stavola, Hydrogen in C-rich Si and the diffusion of vacancy-H complexes, Physica Status Solidi (a). 209 (2012) 1872-1879. 